\title{
Proximity as a Window into the Zone of Proximal Development
}

\author{
Brendan Jacobs, Adam Usher \\ Melbourne Polytechnic, Australia
}

\begin{abstract}
The Zone of Proximal Development (ZPD) is one of the most widely accepted concepts in education but new research using digital technologies has shown that the co-construction of knowledge in the ZPD can also provide a window into the process of conceptual consolidation. This paper uses digital data from primary school children to demonstrate how multimodality can expand the possibilities for projectbased learning in the context of explanatory animation creation.
\end{abstract}

\section{Introduction}

Vygotsky's [18] notion of a ZPD, where learning is extended through working closely with a more experienced helper is one of the most widely accepted theories in education. What is not so well know is that Vygotsky speculated that "what children can do with the assistance of others might be in some sense even more indicative of their [learner] development than what they can do alone” (p. 85). Vygotsky's speculation provides a rationale to further explore the ZPD as a dynamic learning environment where learning occurs, and yet common definitions of the $\mathrm{ZPD}$ [12] reduce it to the difference between what a learner can do without help and what they can achieve with assistance. This distinction is also implied by the most prevalent Venn diagrams of the ZPD where the learner's knowledge and achievement is delineated from the more knowledgeable helper as a small shape within a larger shape. Figure 6 will address and revise this distinction in the Conclusion section.

Much of the discussion around the ZPD involves the complementary notion of 'scaffolding' which looks at the nature of the assistance provided by the more experience helper. Vygotsky never used the term scaffolding as this was introduced by Wood, Bruner and Ross [21] in 1976. Forty years later, Shokouhi and Shakouri [13] suggest that the ZPD is still of great pedagogical interest as "the place where learning and development come together” (p. 61). Valsiner [16] proposed a 'Zone of Free Movement' (ZFM) where limits are placed on a child's options in recognition of the ecological nature of learning and development and the affordances of various stimuli within the natural or staged environment. Valsiner further nuanced the ZFM with the complementary

'Zone of Promoted Actions' (ZPA) as “mechanisms through which the degrees of freedom for the child's actions within environmental settings are selectively regulated" (p. 68).

Valsiner demonstrated the ZFM and ZPA using examples from early childhood where toddlers were taught how to eat with spoons. In such instances these zones were more characteristic of training than conceptual learning. Valsiner had supplemented the ZPD with his ZFM and ZPA but Mercer [10] believed that the ZPD was inadequate and described it as a static concept representing learning at a given point in time. Accordingly, he proposed an 'Intermental Development Zone' (IDZ) as a dynamic learning scenario where the exchange of ideas can lead to conceptual change for both parties. 'Intermental' was used by Mercer to reinforce the notion that the development is between people. In the current study we will argue that the development between people was always inherent in the ZPD and that this relationship can be brought into focus by discussing proximity.

Perhaps the most significant expansion of the ZPD was by John-Steiner [8] when she introduced the notion of a "mutual zone of proximal development" (p. 177) where both parties are learning together as collaborative partners. Collaboration and coconstruction can provide an authentic context for the dynamics of learning by teaching, particularly when digital technologies are introduced. The concept of learning by teaching has been around for thousands of years but Papert [11] saw the potential of technology to enhance this practice, stating that "Learning by doing is an old enough idea, but until recently the narrowness of range of the possible doings severely restricted the implementation of the idea. The educational vocation of the new technology is to remove these restrictions" (p. 22).

Education has a long tradition of embracing technology, but often to use new technology to do the same old things with these new tools. Vygotsky's notion of tool use was intrinsic to his understanding of mediated action, as a tool only becomes such when it is used. As Dron [4] has argued, "a tool separated 
from its use is meaningless: a stick lying in a forest is just a stick” (p. 25). In a recent participatory action research project in Denmark [15] it was found that, while teachers understood the 'ideal' of coconstruction of knowledge and understanding, they saw their 'real' practice as being more aligned with a transmission model than a co-construction reality. Before outlining the particular methods in an animation project with children in Grades 5 and 6, it is necessary to draw on another of Vygotsky's constructs, namely, the dual stimulation method.

\section{Theoretical framework}

Vygotsky and Sakharov's dual stimulation method [19] was used as a theoretical framework to conduct the current study due to the close unity between conceptual tasks and their resolution. The dual stimulation method requires that "the subject must be faced with a task that can only be resolved through the formation of concepts" (p. 124). Vygotsky [19] explained the nature of this link by stating that "the path through which the task is resolved in the experiment corresponds with the actual process of concept formation” (p. 128).

Daniels [3] has defined Vygotsky and Sakharov's dual stimulation method as an experimental approach where people are placed in a situation where "a problem is identified and they are also provided with tools with which to solve the problem or means by which they can construct tools to solve the problem" (p. 822). The first stimulus (i.e., problem) and the second stimulus (i.e., tools) are predetermined and so the point of this method is to understand the effect of the second stimulus on the first. In the current study, the first stimulus was the overall task of explaining a topic, and the second stimulus was the use of the evolving explanatory animation artefact to embody the learning.

As the children were also writing voice-over scripts for their narration, their requests for assistance allowed me to quickly ascertain where they were heading through their explanatory draft material. These interactions seemed to resonate with what Vygotsky [17] described as "tapping the child's thinking” (p. 52). It is this insight into the student's thinking which constitutes the theoretical interest in the current study. As Waldrip and Prain [20] have noted, "Teachers should view representational work by students, including verbal accounts of the topic, as a valuable window into students' thinking and evidence of learning. This assessment can be diagnostic, formative or summative” (p. 27).

Cultural Historical Activity Theory (CHAT) provided both the vocabulary and perspective to understand conceptual change and artefact creation as recursive elements within a collaborative project environment. Although CHAT is based on the work of Lev Vygotsky and his colleagues A. R. Luria and
A. N. Leontiev, of note is Vygotsky's founding contribution through his understanding of mediation and activity through the use of tools. As the children's conceptual task involved multifaceted activities, activity itself became an important issue as it provided a context for this research. Engeström [5] conceptualised CHAT with a focus on knowledge creation stating that "the object of activity is a moving target” (p. 136). Unlike a scientific experiment with clearly defined dependent and independent variables, the children's task in the current study was to determine these variables in relation to their individual topics as evidenced by the scope and sequence of their animations.

In the seminal book Constructionism, digital education pioneers Harel and Papert [6], were amongst the earliest researchers to note that constructing digital artefacts is a multifaceted task. Their insights into this area provided the genesis for the current study as, "The child-producer who wants to design a lesson on the computer must learn about the content, become a tutor, a lesson designer, a pedagogical decision maker, an evaluator, a graphic artist, and so on” (p. 78).

\section{Methods}

Eight boys and girls in grades 5 and 6 participated in an explanatory animation creation project over a period of 17 weeks where they worked on their animation for one hour each week. The children were making these animations for the sake of their own learning and the multimodal nature of their work, combined with the simple practice of saving their work as different date-based files each week, created a digital chronology of their conceptual consolidation embodied in their evolving animation artefacts. The multimodal nature of the data also provided a rationale to present this thesis in its native digital format. All of the data for this research is available online at www.brendanpauljacobs.com.

The animation platform which was used was Microsoft PowerPoint. The children simply inserted auto shapes and then created duplicate slides of each slide (i.e., animation frame) before making incremental changes in position to create the illusion of movement. Saving the work as a series of images (such as JPG) resulted in numbered frames. The images were then combined together using video editing software to render a single video file. The children required some technical help with this process, but they were also given conceptual help for their topics. Co-construction was a major theme in this study which reinforced the nature of proximity within the ZPD as both the student and teacher wrestled with the same pedagogical issues. Although the children were free to choose any topic, 7 out of 8 of the children chose scientific topics as they wanted to know 'how things worked'. Such learning 
dynamics often result in shared learning for both the teacher and student. When both the student and the teacher are learning they could be said to enter a 'mutual zone of proximal development' [8] as collaborative partners. Another feature of this case study was that the children recorded directors' commentaries to document their decision-making processes. This provided a window into their own learning and encouraged metacognition in tangible ways as the children's voices could be heard discussing their pedagogical decision-making processes and aesthetic choices.

\section{Data analysis and results}

Summaries of the learning journeys for two of the eight participants are presented here to exemplify the complexity and multimodal nature of the explanatory animation process and the creation of mutual ZPDs with the primary researcher (who shall, in sections 4 and 5, be referred to in first person).

Analysing the progress of each student was an ongoing process where I sought to offer specific help for particular challenges throughout the 17 weeks of the project. Although some technical animation help was required, my primary role was in relation to the conceptual questions which the students had about their own content knowledge. Of the twelve data sources that were collected, two were used for ongoing comparison:

1. What each student could articulate about their topic through their voice-over script.

2. What I knew about the topic as documented through my weekly reflections.

The grounds for comparison (i.e., why compare the children's voice-over scripts and my researcher reflections?), involve the notion that storyboards are semiotic tools for cross-modal cognition [7]. The term 'cross-modal cognition' describes the ways in which learners in a multimodal environment creating explanatory animations are simultaneously working with different modalities, such as images and words, as different aspects of the same pedagogical task. The co-construction of knowledge as evidenced through the evolving digital artefacts also surfaced my own understanding of the topic. This provided a logical context for analysis as I would not have been able to make any judgements about each student's work without reference to my own understanding [9].

The iterative nature of learning is consistent with Vygotsky's [18] notion of a ZPD where a more experienced or knowledgeable helper, such as a teacher or parent, can provide targeted and personalised assistance to another person to facilitate learning. In the study reported here, the ZPD formed the frame of reference for ongoing comparisons between what each student knew about their chosen topic and what I knew as their helper. The ZPD is usually understood as a context for teaching and learning rather than a framework for analysis but this approach is not without precedent as Sutter [14], has also noted the connection between the ZPD and Vygotsky and Sakharov's dual stimulation method.

Extracts from two out of the eight participants are presented here as examples of proximity within mutual ZPD. These results involve students with the pseudonyms 'Neil' and 'Harriet'.

\section{Neil "Satellites"}

Neil was a Grade 6 boy who chose to investigate satellites. (Neil's animation is available online at www.brendanpauljacobs.com/satellitesreview.html). In his prior knowledge video, Neil stated that "My topic is satellites and I really don't know much about them except they're used for transmitting signals to devices around us, say, for cable TV, or, umm, GPSs and, so, yeah. That's all I know about them”. Neil began his animation cautiously and often checked with me to see that he was doing the right thing as noted in my research reflection at the time:

Neil seemed to be very concerned that he complied with my guidelines as he often asked; "Can I do this?" I have since reassured Neil that he is free to follow his own path as it is his learning and decision-making processes in which I am most interested.

This cautionary approach changed dramatically after the first session once Neil understood that he didn't need my approval. The "head start" that Neil referred to in his director's commentary was that he immediately grasped the concept of the iterative PowerPoint animation technique for "Insert duplicate slide". I had a constant struggle with Neil from this point forward to get him to complete a key frame before proceeding to create the movement sequences. Otherwise, Neil would end up double handling all of his imagery as each correction or improvement would have to be implemented multiple times.

Satellites seemed to be a great topic for an animation as the visual and spatial possibilities of showing the Earth as a globe appeared to be obvious. Neil commenced the project by stating in his prior knowledge video that satellites are "used for transmitting signals to devices around us, say, for cable TV, or, umm, GPSs”. The next logical step for Neil was to move beyond what satellites do so that he could investigate how satellites work.

As with many of the children in the study, asking the right questions was an important part of guiding their progress. The question that moved Neil into the ZPD was, "How many satellites are needed to transmit a signal around the world?" My reason for posing this question to Neil was that I wanted him to understand that satellite signals travel in straight lines. The noted science fiction author Arthur C. Clarke [2] answered his own hypothetical question about this issue in 1945 [several years before satellites were even invented] stating that "three satellite stations would ensure 
complete coverage of the globe" (p. 306). Based on this insight, it was logical to proceed with the planetary, Earth imagery as shown in Figure 1.

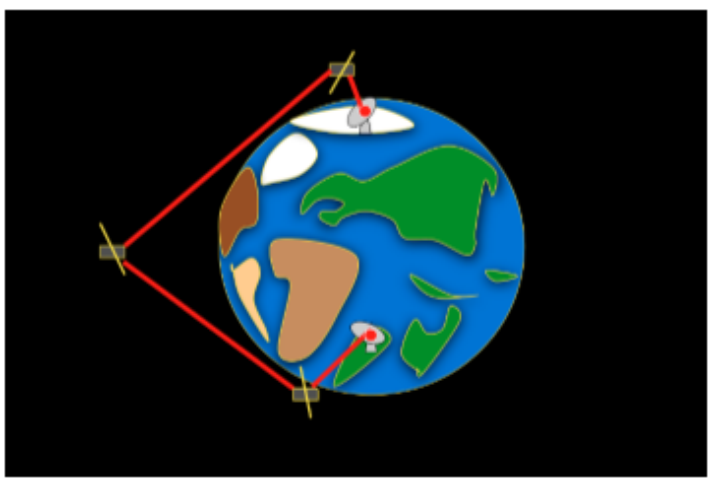

Figure 1. Screen shot of a satellite signal

Neil introduced his space imagery with an octagon metaphor to illustrate his statement that "satellites can only send transmissions in straight lines but the Earth is round”. Animating the satellite transmission lines smoothly proved to be impractical in PowerPoint because it wasn't possible to make small, incremental changes between each frame. Any disruption to the position of the lines was unacceptable so we got around this by drawing a completed transmission signal (i.e., red line) and then erasing small parts of the line in Adobe Photoshop. Each change was then "Saved as" a new file and then these files were played in reverse order to show the transmission signal moving and expanding. Although Figure 1 was an actual screen shot, it also provided the source material for all of the preceding animation frames using this reverseengineering approach. Neil began a tangent about satellite protocols during Session 8:

So today I did a bit of research and I found out that a satellite, say one company's satellite, can't send a transmission to a different company's satellite 'cause they need to have, umm, the same program (or something like that) to get it to where it needs to go. I'm going to make a, some more slides that, I'm going to put in a new coloured satellite. And I'm going to have one of my satellites send a signal to it and then it comes up with a red cross on the new satellite and it sends it back.

I encouraged Neil to abandon this issue because I considered this to be additional information that would make his animation too long. I likened the compatibility issue to different cell phones using different networks and how the differences probably relate to settings, configurations and company or country protocols rather than true functionality. Neil agreed to abandon the compatibility issue but this discussion proved to be useful to help determine what is essential information for this topic.

Having described and depicted the satellite transmission signal path, the final issue to be covered related to what actually happens when a signal is received and then transmitted to another satellite. Neil addressed this explicitly in his voice-over script stating that "Inside a satellite is a transponder which changes the frequency and amplifies the signal before sending it on". When we learnt that "transponder" was a portmanteau (i.e., a merging of two words), the visual potential for morphing transmitter and responder together was obvious. Figure 2 shows the two words as they start to merge.

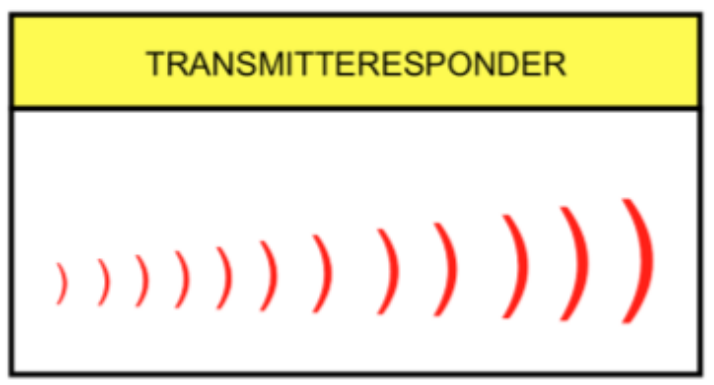

Figure 2. Screen shot of the transponder portmanteau

This simple device was very helpful because it would have been extremely difficult to actually show how the signal's frequency is changed inside the transponder. The actual sequence was:

\section{TRANSMITTER RESPONDER \\ TRANSMITTERESPONDER \\ TRANSMITTEESPONDER \\ TRANSMITTESPONDER \\ TRANSMITESPONDER \\ TRANSMITSPONDER \\ TRANSMISPONDER \\ TRANSMIPONDER \\ TRANSMPONDER \\ TRANSPONDER}

The need to be able to visualise the invisible satellite signals provided additional opportunities for us to discuss the properties of these transmissions. Neil's early attempts at representing a satellite signal involved moving brackets [ ())))) ] which resembled a ripple on a pond. I suggested that he use a lengthening line [ _ _ ] to show that the signals are constant rather than intermittent. Neil was agreeable but somewhat annoyed that he would need to recreate some of his imagery.

Neil clearly enjoyed the animation process. I often wondered if he was more interested in creating movement than learning about his topic. I asked Neil to elaborate on his learning during the debriefing session on the last day of the project:

Brendan: How do you think you learnt compared to if you did something that wasn't on a computer. Like if you were making posters or writing a normal sort of assignment? Do you think you would have 
learnt as much or do you think it was...do you think this was a, a better or worse way to go about it?

Neil: I think like a visual presentation like on a computer, you can just explain it more and it's more entertaining and more like...you, you have a lot more ways to go about it where...whether...if you do it on like a poster or a piece of paper, I think your choices would be much, much more limited in how you'd want to do it or set it out.

Neil's enthusiasm often turned into frustration as he rarely heeded my advice to get his imagery correct before creating movement through the "Insert duplicate slide" process. At one stage, Neil thought that he had finished but there were issues with his imagery that we still had to resolve. His summary at this time was that, "Today I actually found out that I haven't finished, according to Brendan, which I'm not happy with". The particular issue related to the background colour of outer space. Neil was using white rather than black and he had also applied a shadow. Figure 3 is a discarded screen shot from a complete series of 89 frames.

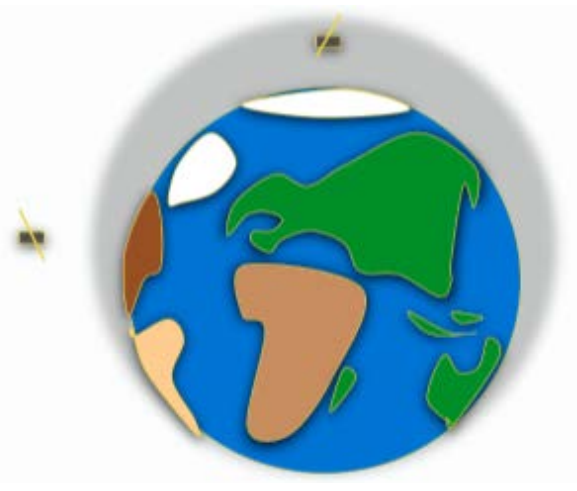

Figure 3. Discarded animation imagery

Neil was annoyed about having to redo this, but, once completed, he could see that his animation was more realistic using black for outer space. During the last session, there was still work to be done so Neil demonstrated his own sense of agency by delegated these finishing touches to me: "I've instructed Brendan and told him what I need to be done and he's going to be helping me finish it which is really good". I asked Neil how he felt about the issue of coauthorship during the group's debriefing session:

Brendan: How did you feel about me working on your work? Do you think it took away from it being yours as much or were you just happy that you had help?

Neil: Well, I think I really appreciate that Brendan could help me and I just, it just felt like it took away a bit of the pressure and I don't feel that... as if it...my project was being taken away by me. I still think, Brendan helped out a lot but I still did a lot, umm, with it. And he just helped me and just guided me as well and yeah and I really appreciate that.
Neil developed a deep understanding of everything he presented in his animation. There were, however, some issues that we left out make the topic more manageable such as the geosynchronous orbit that keeps each satellite in position. If Neil was to start another animation now he would be much more efficient as he understands the importance of building appropriate imagery before attempting to animate it. The interactions between Neil and myself alternated between specific content related to satellites, pedagogical decisions about the animation sequences, and technical animation matters. All three areas were interelated but it was the coconstruction of knowledge through the ZPD which charactised our interactions.

\section{Harriet - "How does hair grow?"}

Harriet was a Grade 6 girl who chose to investigate how hair grows. (Harriet's animation is available online at www.brendanpauljacobs.com/hairreview.html). She stated in her prior knowledge video that "My topic is how hair grows and I don't really know much about it except that it's from your skull and there's a tiny stem inside your skull and it grows from there”.

Harriet was the most independent child out of all of the eight participants. In many ways, Harriet was the model student as she was diligent, focussed, engaged and making steady progress, only seeking my assistance for technical animation advice rather that specific content knowledge. She noted in her weekly reflections that I asked her questions to keep her learning. One of my first questions was "Is hair dead or alive?” Harriet addressed this in the opening scene of her animation:

"Is hair dead or alive?" A lot of people ask that but the truth is...hair is dead and that's why it doesn't hurt when you cut it. But the reason it does hurt when you pull it is because you're also pulling the stem and that's where the hair grows from.

These questions helped to guide Harriet's learning as she began to seek more detail about the structure of hair. One of my suggestions was that Harriet should create some cross-sectional imagery. Harriet proceeded to identify the various components of hair in the voice-over script of her animation as follows:

There are many different components that make up the hair.

- the skin around the skull which is also known as the scalp

- the hair string

- the sebaceous glands

- the dermal papillae and

- the hair shaft.

Harriet had encountered some information that I had never heard of such as 'sebaceous glands'. She appeared to be engaged and eager to research new 
terminology. As such, Harriet essentially directed her own progress. I was grateful for Harriet's selfmotivation, as there was always at least one of the other children with their hand up, wanting help.

I have continued to reflect on Harriet's work since completing the data collection. My most recent reflection is that the topic that Harriet presented could have been more accurately described as "What is hair made of?" To further explore the system qualities of hair growth, we should have looked into the hair growth cycle. I have now discovered that there are three distinct stages of the hair growth cycle, which is significant because Harriet and I never encountered any of these stages during the project. The absence of this key information accounts for our failure to identify the system qualities of hair growth. These three stages could also be called phases as the Oxford English Dictionary defines a phase as "a particular stage in recurring sequence of movements or changes". The three phases of hair growth are

1. Anagen (active phase lasting between 2 - 6 years)

2. Catagen (transitional phase lasting around 2 weeks)

3. Telogen (resting phase lasting between 1 - 4 months)

Harriet's failure to identify the three phases of hair growth was really my own failure to ask her the right questions such as "What is the hair growth cycle?" I would have quickly discovered these terms if I had done any of my own research but this was the one case where a child did all of their own research. This further caused me to reflect on my role as a teacher working in a primary school. The old adage the squeaky wheel gets the grease is characteristic of the way that the most demanding children usually receive the most assistance from their teacher. Since this time I have sought to create opportunities to extend the learning of the more independent children with suitable questions and challenges.

Harriet's progress was unlike any of the other seven children as her animation could be characterised as an animated poster with annotated diagrams and corresponding narration, rather than an explanatory narrative. This is because Harriet's voice-over script described components rather than explaining relationships between them or the cycle to which they belonged as follows:

So the hair grows on and on so slowly that you don't even notice it. It grows and falls out at the same time but because it grows so fast, people can choose to have long hair. Now the reason that people have different coloured hair and different

types of hair like curly, straight or wavy is because of a little something called...genes.

Harriet wrote in her Director's commentary that "Genes was my answer to different colours and curly, straight and wavy but I didn't really get into any of the details because that's not my topic”. Andreou [1] calls this a graphical metaphor when words or symbols are "arranged in meaningful spatial configurations that metaphorically (or allegorically) express relations among the concepts [or] the objects" (p. 15) as depicted in Figure 4:

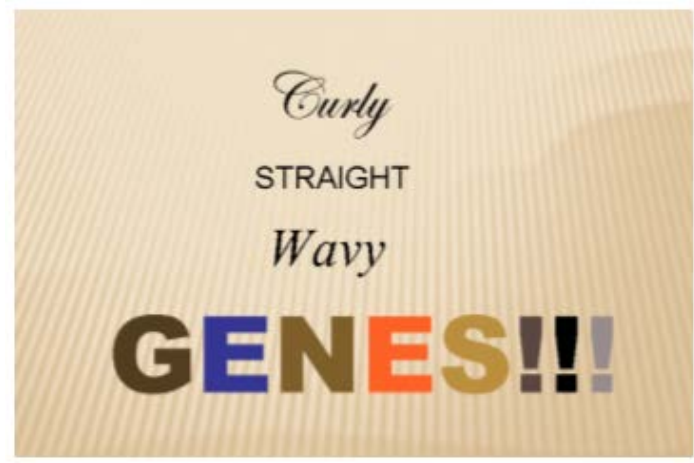

Figure 4. A graphical metaphor of the attributes of hair

Harriet's case is unique as she was the only child amongst the eight participants who didn't use a metaphor to explain her topic. In retrospect, the cyclical nature of Harriet's topic escaped both Harriet and myself.

\section{Discussion}

As this article emphasises proximity in the ZPD, it could be inferred that merely the teacher's presence is a catalyst for learning, but anyone who has ever set foot in a classroom knows that this isn't necessarily the case. Harriet's animation was the least effective in terms of her own conceptual growth and yet I had characterised her as the ideal student. This was because her gradual acquisition of facts did not require her to wrestle with pedagogical issues at a conceptual crossroads where higher-level learning could occur. I know this to be true of my own experiences in these encounters with Harriet as we never had to wrestle with conceptual issues.

Proximity alone did little to promote Harriet's learning because there was no co-construction occurring, which appears to be the missing element in Harriet's conceptual journey. Possible metaphors to demonstrate the iterative nature of the hair growth cycle could have been grass growing, or finger nails growing. I'm confident that we would have discovered these phases if we had amended the title to be "The hair growth cycle". The hair growth cycle would have lent itself to animation as the duration of the anagen stage explains why some people can grow longer hair than other people.

These examples from Neil and Harriet illustrate aspects of the ZPD and some key premises of the dual 
stimulation method. One premise is that the second stimulus is used as a tool to resolve the first stimulus. The unity between task and tool was further developed by Vygotsky [18] when he stated that the dual stimulation method is "simultaneously prerequisite and product, the tool and result of study" (p. 65).

Another premise is that the specific path of the resolution throughout the first stimulus (established by the second stimulus) will chronicle the formation of concepts [19]. The conceptual journeys within each portrait were chronological in relation to the various problem-solving events. This notion of conceptual evolution has been further developed by Daniels [3] who noted that the historicity of development is an importantly component of the dual stimulation method.

In the current study, conceptual consolidation was also understood as a history or chronology of development. An affordance of the dual stimulation method was that it created both the conditions for conceptual change and also provided the means to document conceptual change. This was achieved through the evolving, date-based multimodal animation artefacts and the proximity afforded to me as the researcher through the ZPD.

Figure 5 represents a revision of the ZPD with the overlapping red spikes where the child's development surpasses the helper. This was inspired by my work with Harriet, particularly near the beginning of the project where she started using terminology that I had never heard of.

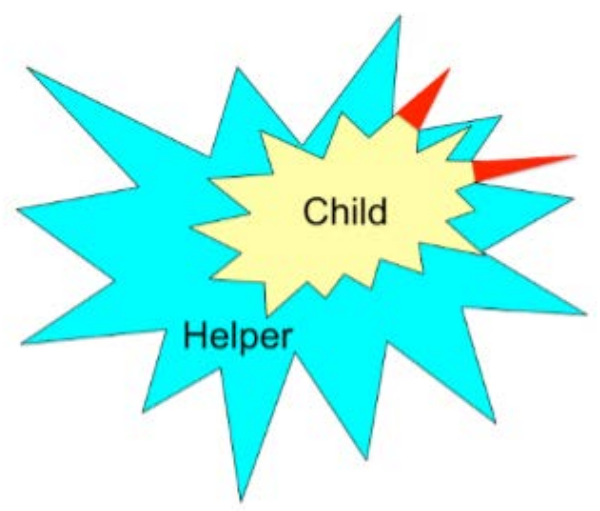

Figure 5. Revising the ZPD

\section{Conclusion}

It is clear that the critical commonality between Neil and Harriet was not the newness of the technology, but the use of technology as a tool to make visible the form and purpose of the activity. When students construct multimodal artefacts for the sake of their own learning, the power of the explanatory animation creation process is its ability to track and illustrate the conceptual-developmental pathway.

Opportunities for meaningful interactions revolved around the children's emerging representations available on their computer screens, stimulating discussion and critique. The children's activity was seen to segue between different roles at various times, often within the same session as they were working on multifaceted tasks. Seven distinct activities constituted the explanatory animation creation task, namely:
1. Researcher
2. Graphic artist
3. Script writer
4. Narrator
5. Animator
6. Video editor
7. Pedagogical decision maker

The alignment between purpose and activity illuminates not only the resolution of the issue, but also the connection between the students to themselves as learners. This is where formative assessment data abounds, particularly when working in the digital realm as every iteration of a digital project is preserved and easily accessible for further critique.

Some of the greatest educational theorists of the past century such as Vygotsky and Dewey have noted how concepts develop over time and that they are not immediately grasped by the learner. Jacobs, Wright and Reynolds [7] suggested that cross-modal cognition occurs on a continuum between abstract and concrete as ideas become increasingly concrete as they become consolidated. Accordingly, we present Figure 6 [as an improvement over Figure [5] as a way to understand the ZPD nuanced with Vygotsky's insight that concepts develop over time:

The broken lines represent learning that has yet to become consolidated and the solid lines represent consolidated learning. The incongruence of the child and helper shapes indicates that both and are in a mutual ZPD and that the child's knowledge can exceed that of the helper in certain instances.

Lifelong learning is often heralded as a hallmark of education, but it must be asked whether we as educators actually see co-construction as part of this reality. This question should also help to orientate us as to whether we are primarily seeking formative or summative assessments. Co-construction is at the heart of a mutual ZPD as formative assessment whereas standardised testing is typically summative. In our experience, reintroducing choice to students is a key to engaging them in their own learning, which, to use another famous term from Vygotsky, enables the student to stand "a head taller" [18]. In light of the opportunities afforded by digital technologies, it is our 
hope that the prevailing learning mindset and practices described in this case study can serve as a rationale for focusing on learning rather than achievement.

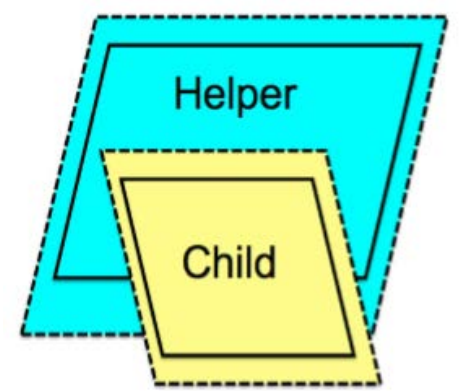

Figure 6. Learning in a mutual ZPD

The acquisition of knowledge and skills is meaningless if they are not given an authentic purpose within a learning context or a learning 'construction zone'.

\section{References}

[1] Andreou, A. P. (2013, October). Conceptual metaphors as image schemas in information visualizations. In 2CO Communicating complexity: 2013 Conference Proceedings (pp. 12-18). Edizioni Nuova Cultura, University of Sassari, Italy.

[2] Clarke, A. C. (1945, October). Extra-terrestrial relays Can rocket stations give worldwide radio coverage? Wireless World, 305-308.

[3] Daniels, H. (2012). The interface between the sociology of practice and the analysis of talk in the study of change in educational settings. In J. Valsiner (Ed.) The Oxford handbook of culture and psychology (pp. 817- 829). New York, NY: Oxford University Press.

[4] Dron, J. (2012). The pedagogical-technological divide and the elephant in the room. International Journal on ELearning, 11(1), 23-38.

[5] Engeström, Y. (2001). Expansive learning at work: Toward an activity theoretical reconceptualisation. Journal of Education and Work, 14(1), 133-155.

[6] Harel, I. \& Papert, S. (Eds.) (1991). Constructionism. Norwood, NJ: Ablex Publishing Corporation.

[7] Jacobs, B., Wright, S. \& Reynolds, N. (2017). Reevaluating the concrete - Explanatory animation creation as a catalyst for cross-modal cognition. Mind, Culture, and Activity. http://dx.doi.org/10.1080/10749039.2017.1294181

[8] John-Steiner, V. (2000). Creative collaboration. New York, NY: Oxford University Press.

[9] Leite, L., Mendoza, J. \& Borsese, A. (2007). Teachers' and prospective teachers' explanations of liquid-state phenomena: A comparative study involving three European countries. Journal of Research in Science Teaching, 44(2), 349-374.

[10] Mercer, N. (2008). The seeds of time: Why classroom dialogue needs a temporal analysis. Journal of the Learning Sciences, 17(1), 33-59.

[11] Papert, S. (1991). Perestroika and epistemological politics. In I. Harel \& S. Papert, S. (Eds.) Constructionism (pp. 13-28). Norwood, NJ: Ablex Publishing Corporation.

[12] Reber, A. S., Allen, R. \& Reber, E. S. (1995). The Penguin dictionary of psychology (4th ed.). London, UK: Penguin Books Ltd.

[13] Shokouhi, M. \& Shakouri, N. (2015). Revisiting Vygotsky's concept of zone of proximal development: Towards a stage of proximity. International Journal of English Literature and Culture, 3(2), 60-63.

[14] Sutter, B. (2001). Instruction at heart: Activitytheoretical studies of learning and development in coronary clinical work [Doctoral dissertation]. Karlskrona, SE: Blekinge Institute of Technology.

[15] Usher, A. (2017) Creating teachers as scholars: Meeting the challenges of $21 \mathrm{C}$ globalized education. [PhD thesis]. Melbourne, Vic: Victoria University. Retrieved from http://vuir.vu.edu.au/id/eprint/35049

[16] Valsiner, J. (1984). Construction of the zone of proximal development in adult child joint action: The socialization of meals. New Directions for Child and Adolescent Development, 1984(23), 65-76.

[17] Vygotsky, L. (1962). Thought and language. Cambridge, MA: The M.I.T. Press.

[18] Vygotsky, L. (1978). Mind in society: The development of higher psychological processes. Cambridge, MA: Harvard University Press.

[19] Vygotsky, L. (1987). Collected works of L.S. Vygotsky (Vol. 1). R. W. Rieber \& A. S. Carton (Eds.), New York, NY: Plenum Press.

[20] Waldrip, B. \& Prain, V. (2013). Teachers' initial response to a representational focus. In R. Tytler, V. Prain, P. Hubber \& B. Waldrip (Eds.), Constructing representations to learn in science (pp. 15-30), Rotterdam, NL: Sense Publishers.

[21] Wood, D. J., Bruner, J. S. \& Ross, G. (1976). The role of tutoring in problem solving. Journal of Child Psychology and Psychiatry, 17(2), 89-100. 